\title{
GALACTIC PERTURBATIONS INFLUENCE ON OBSERVABILITY OF INTERSTELLAR COMETS
}

\author{
H. PRȨTKA \\ Astronomical Observatory, A. Mickiewicz University \\ Poznań, Poland
}

\begin{abstract}
Some cases of the cometary evolution from the interstellar space to the observability region due to the galactic disk perturbations are shown. Simulated parameters of potentially observable interstellar comets are presented.
\end{abstract}

\section{Introduction}

The infrared observations of selected nearby stars (Beta Pictoris was the first) show that a majority of ordinary main-sequence stars may be surrounded by a kind of "cometary cloud", similar to the Oort Cloud that was proposed to exist around the Solar system (Oort, 1950). Comets can originate in the process of forming stars and their planetary systems from protostellar nebulae and then they are mostly ejected into the interstellar space by planetary and stellar perturbations (Weissman, 1990). Alternatively, they can also originate directly from the interstellar medium (Valtonen and Innanen, 1982). It means that we could imagine a large number of comets migrating through interstellar space and occasionally being captured by the Solar system. Thus, we may ask: is it possible to find interstellar comets in the population of observed near-parabolic comets (Yabushita, 1991)? An extensive discussion of various ideas on the cometary origin can be found in the book: 'Origin of comets' by Bailey, Clube and Napier (1990).

\section{Dynamics of the Problem}

This paper presents the initial stage of a project begun in Poznan Observatory a year ago. In this work, we consider the possibility that galactic perturbations can change the orbit of an interstellar comet and direct it into the inner part of the Solar system. By "interstellar comets" we understand both comets on hyperbolic orbits and those on extremely elongated elliptic orbits. They are formally bound to the Sun but with aphelia far beyond the

I. M. Wytrzyszczak, J. H. Lieske and R. A. Feldman (eds.), Dynamics and Astrometry of Natural and Artificial Celestial Bodies, 155, 1997.

(C) 1997 Kluwer Academic Publishers. Printed in the Netherlands. 
conventional limit of the Oort Cloud typically assumed to be smaller than $160000 \mathrm{AU}$ in diameter. In the first step, we restrict our dynamical model to the influence of the Sun and the Galactic disk, omitting perturbations caused by planets, stars and the Galactic centre.

The motion of the comet is described by the following equations (Heisler and Tremaine, 1986; see also: Dybczyński and Prętka, 1996):

$$
\ddot{x}=-\frac{\mu}{r^{3}} x, \quad \ddot{y}=-\frac{\mu}{r^{3}} y, \quad \ddot{z}=-\frac{\mu}{r^{3}} z-4 \pi G \rho \cdot z
$$

where $x, y, z$ are the rectangular coordinates of a comet on the galactic reference frame, $4 \pi G \rho \cdot z$ describes the disturbing force perpendicular to the galactic disk and $\rho$ is the local matter density in the Solar neighbourhood, equal to $0.185 M_{\odot} / p c^{3}$ (Bahcall, 1984). The equations of motion are numerically integrated and the trajectory of the comet is studied. Careful examination of several analytical and semi-analytical methods of solving these equations shows that at large distances from the Sun their validity fails and the only efficient method of studying an individual orbital path is the direct numerical integration of these equations in rectangular coordinates (Dybczyński and Prętka, this volume; see also Breiter, Dybczyński and Elipe 1996).

\section{Results}

We present here two examples of the motion of a simulated observable interstellar comet under the influence of the galactic disk tides. Each example consists of a figure and a table of data. The figures show the projection of the orbital path onto the plane perpendicular to the galactic disk plane, the tables show the list of passages of a comet through the planetary region. The trajectories of comets coming from the interstellar space to the vicinity of the Sun are drawn, starting at an arbitrary moment of time. At the bottom of each plot its scale is compared to the size of the classical Oort cloud. The energy of the comets are calculated from the following formula (Heisler and Tremaine, 1986):

$$
E=-\frac{\mu}{r}+\frac{V^{2}}{2}+2 \pi G \rho \cdot z^{2}
$$

and expressed in inverse astronomical units $\left(A U^{-1}\right)$ to be compared with the energy of the comet in a keplerian orbit. The comets we investigate have energies in the interval $\left(-10^{-6} ;+10^{-6}\right) A U^{-1}$. The first plot is drawn for a comet formally bound to the Sun (with positive energy) and the second one is for an comet unbound (negative energy), i.e. coming from interstellar space. In both cases the comets come to the Sun from a distance much greater than the outer limit of the Oort cloud, and in both cases their trajectories are similar. Such comets may pass many times through the inner part of the Solar system where they can be observed. 


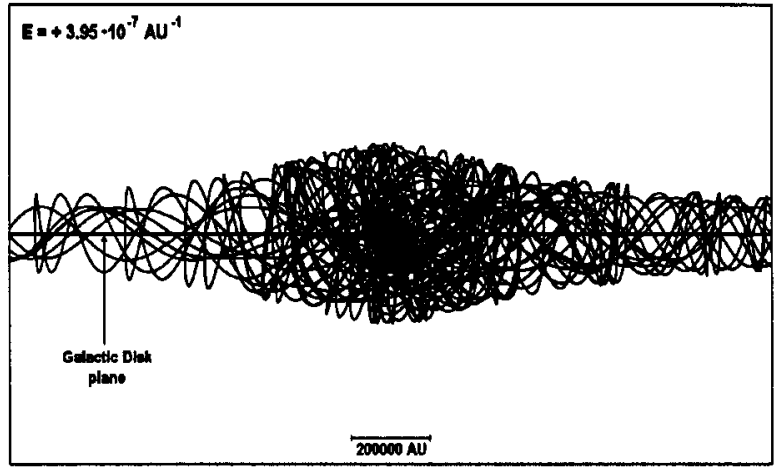

Figure 1. Trajectory of the comet bound to the Sun in the plane perpendicular to the galactic disk.

TABLE 1. Osculating orbital elements recorded at the moment when the comet enters the planetary region (for the comet bound to the Sun).

\begin{tabular}{cccc}
\hline perihelion distance [AU] & eccentricity & argument of perihelion $\left[{ }^{\circ}\right.$ ] & inclination $\left[^{\circ}\right]$ \\
\hline 3.15 & 0.99999876 & 271.9 & 80.3 \\
12.50 & 0.99999506 & 50.5 & 85.2 \\
5.2 & 0.99999792 & 4.3 & 82.5 \\
12.11 & 0.99999521 & 11.1 & 85.1 \\
0.35 & 0.99999986 & 351.6 & 59.6 \\
4.75 & 0.99999812 & 14.0 & 82.1 \\
1.18 & 0.99999953 & 121.5 & 74.0 \\
\hline
\end{tabular}

The tables show the list of osculating orbital elements recorded at the moment when the comet enters the region of planetary perturbations (closer than $50 \mathrm{AU}$ ). All angular elements are measured with respect to the Galactic disk plane.

From the examples presented we can see that the Galactic disk tidal force can occasionally direct interstellar comets into the vicinity of the Sun and into the region of observability. Additionally, the values of the osculating eccentricity of the orbit noted at the moment of perihelion passage are comparable with the values of eccentricities of the observed near-parabolic comets.

At this initial stage of the project we neglect planetary perturbations and the influence of the Galactic centre. The first will be added in the simulation programme to calculate the statistical characteristics of the population of observable interstellar comets. Three different methods are currently being tested and compared to choose the most efficient and accurate one. 


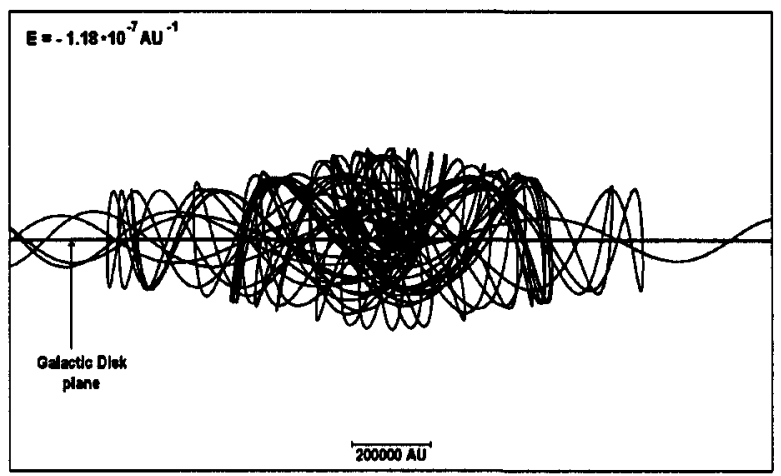

Figure 2. Trajectory of the comet unbound to the Sun in the plane perpendicular to the galactic disk.

TABLE 2. Osculating orbital elements recorded at the moment when the comet enters the planetary region (for the unbound comet).

\begin{tabular}{cccc}
\hline perihelion distance $[\mathrm{AU}]$ & eccentricity & argument of perihelion $\left[{ }^{\circ}\right]$ & inclination $\left[{ }^{\circ}\right]$ \\
\hline 4.61 & 1.00000005 & 77.0 & 76.5 \\
45.0 & 1.00000053 & 319.2 & 85.7 \\
34.76 & 1.00000041 & 211.9 & 85.1 \\
8.02 & 1.00000009 & 220.4 & 79.8 \\
\hline
\end{tabular}

Acknowledgements. The work was supported by the grant No 2.P03D.024.10 of Polish Committee of Scientific Research.

\section{References}

Bailey, M.E., Clube, S.V.M., and Napier, W.M.: 1990, The Origin of Comets, Pergamon Press.

Breiter, S., Dybczyński, P.A., and Elipe, A.: 1996, "The action of the Galactic disk on the Oort cloud comets. Qualitative study", Astron. Astrophys., in press.

Bahcall, J.N.: 1984, "Self-consistent determinations of the total amount of matter near the Sun", Astrophys. J. 276, 169-181.

Dybczyński, P.A. and Prętka, H.: 1996, "The statistical effect of galactic tides on the Oort cloud", Earth, Moon and Planets 72, 13-18.

Dybczyński, P.A. and Prętka, H.: 1997, "The Galactic disk tidal force: Simulating the observed Oort cloud comets", this volume.

Heisler, J. and Tremaine, S.: 1986, "The influence of the galactic tidal field on the Oort comet cloud", Icarus 65, 13-26.

Oort, J.H.: 1950, "The structure of the cloud of comets surrounding the Solar system, and the hypothesis concerning its origin", Bull. Astron. Inst. Nether. 11, 91-110.

Valtonen, M.J. and Innanen, K.A.: 1982, "The capture of interstellar comets", Astrophys. J. 225, 307-315.

Yabushita, S.: 1991, "Maximum non-gravitational acceleration due to out-gassing cometary nuclei", Earth, Moon and Planets 52, 87. 